\section{(6) OPEN ACCESS}

\title{
Acute kidney injury is independently associated with death in patients with cirrhosis
}

\author{
Robert A Scott, ${ }^{1}$ Andrew S Austin, ${ }^{1}$ Nitin V Kolhe, ${ }^{2}$ Chris W McIntyre, ${ }^{2}$ \\ Nicholas M Selby ${ }^{2}$
}

${ }^{1}$ Department of Gastroenterology and Hepatology, Derby Digestive Diseases Centre, Royal Derby Hospital, Derby, Derbyshire, UK ${ }^{2}$ Department of Renal Medicine, Royal Derby Hospital, Derby, UK

\section{Correspondence to} Dr N Selby, Department of Renal Medicine, Royal Derby Hospital, Uttoxeter Road, Derby, DE22 3NE, UK; nick.selby@nhs.net

Received 3 December 2012 Revised 6 March 2013 Accepted 9 March 2013 Published Online First 18 April 2013
To cite: Scott RA, Austin AS, Kolhe NV, et al. Frontline Gastroenterology 2013:4:191-197.

\section{ABSTRACT}

Background and aims Current creatine-based criteria for defining acute kidney injury (AKI) are validated in general hospitalised patients but their application to cirrhotics (who are younger and have reduced muscle mass) is less certain. We aimed to evaluate current definitions of AKI (acute kidney injury network (AKIN) criteria) in a population of cirrhotic patients and correlate this with outcomes.

Methods We prospectively identified patients with AKI and clinical, radiological or histological evidence of cirrhosis. We compared them with a control group with evidence of cirrhosis and no AKI.

Results 162 cirrhotic patients were studied with a mean age of $56.8 \pm 14$ years. They were predominantly male $(65.4 \%)$ with alcoholic liver disease (78.4\%). 110 patients had AKI: 44 stage 1, 32 stage 2 and 34 stage 3 . They were well matched in age, sex and liver disease severity with 52 cirrhotics without AKI. AKI was associated with increased mortality $(31.8 \%$ vs $3.8 \%, p<0.001)$. Mortality increased with each AKI stage; $3.8 \%$ in cirrhotics without $\mathrm{AKI}$, $13.5 \%$ stage $1,37.8 \%$ stage 2 and $43.2 \%$ stage 3 ( $p<0.001$ for trend). Worsening liver disease (Child-Pugh class) correlated with increased mortality: $3.1 \%$ class A, $23.6 \%$ class B and $32.8 \%$ class $C$ ( $p=0.006$ for trend). AKI was associated with increased length of stay: median 6.0 days (IQR 4.0-8.75) versus 16.0 days (IQR 6.0-27.5), $\mathrm{p}<0.001$. Multivariate analysis identified $A K I$ and Child-Pugh classes B and $C$ as independent factors associated with mortality.

Conclusions The utility of AKIN criteria is maintained in cirrhotic patients.

Decompensated liver disease and AKI appear to be independent variables predicting death in cirrhotics.

\section{INTRODUCTION}

Mortality from liver disease is increasing. ${ }^{1}$ Key drivers of this increase are the rising prevalence of alcoholic liver disease, viral hepatitis and fatty liver disease. ${ }^{2}$ Liver disease is now the fifth highest cause of death in the UK, with a $25 \%$ increase in mortality between 2001 and 2009. ${ }^{3}$ Renal dysfunction is a common complication of cirrhosis and confers a poor prognosis. ${ }^{4}$ Renal dysfunction occurs in 20\% of patients with cirrhosis admitted to hospital, often linked with other complications of cirrhosis such as variceal bleeding and spontaneous bacterial peritonitis. ${ }^{5}$ In a systematic review of 118 studies, the presence of renal dysfunction was a powerful predictor of death in decompensated cirrhosis. ${ }^{6}$ Hepatorenal syndrome (HRS) has an extremely poor prognosis with a median survival time of 3 months, ${ }^{7}$ falling to just 1 month in those with untreated Type 1 HRS. $^{8}$ Serum creatine is one of three variables comprising the model of end-stage liver disease score which is widely used in predicting short term mortality in allocating priority for orthotopic liver transplantation. ${ }^{9}$

Many definitions of renal dysfunction in liver disease are based on creatine thresholds, such as those used in HRS classification. ${ }^{10}$ This approach does not account for individual variation in baseline renal function or that a significant decline in renal function can occur with much smaller changes in creatine. There has also been a lack of standardisation in defining these thresholds. ${ }^{10-13}$ A more evolved approach to defining acute renal dysfunction has come with the widespread acceptance of consensus criteria for acute kidney injury 
(AKI) that provide a method of diagnosing and describing the severity of renal dysfunction based on individualised changes in serum creatine and urine output. The Kidney Disease: Improving Global Outcomes (KDIGO) diagnostic criteria are the most current, ${ }^{14}$ combining the earlier acute kidney injury network (AKIN $)^{15}$ and Risk of renal dysfunction; Injury to the kidney; Failure of kidney function; Loss of kidney function; and End-stage kidney disease (RIFLE) classifications. ${ }^{16}$ The use of these criteria has been validated in a variety of settings including the critically ill and general hospitalised patients. ${ }^{17-21}$ Importantly, these criteria recognise that even a small decline in renal function is associated with poor outcomes. ${ }^{22}$ AKI therefore encompasses a wide spectrum of illness in a large number of patients from an abrupt rise in serum creatine of only $26.4 \mu \mathrm{mol} / \mathrm{l}(0.3 \mathrm{mg} / \mathrm{dl})$ to critically unwell patients requiring renal replacement therapy.

However, there are several reasons why creatine-based definitions of AKI may perform differently in patients with liver disease. Cirrhotics are younger and have reduced muscle mass compared with other hospitalised patients. ${ }^{23}$ Severe hyperbilirubinaemia gives a falsely low value of serum creatine with chemical rather than the enzymatic measurement techniques. ${ }^{24}$ These factors may result in lower than expected baseline creatine values in this patient group relative to glomerular filtration rate. ${ }^{25} \mathrm{~A}$ small number of studies have begun to evaluate the current AKI criteria in cirrhotics. Some have focused on specific groups, such as those in intensive care or decompensated patients with ascites, which may limit their generalisability. ${ }^{26-28}$ The only prospective trial using robust AKIN definitions in a cirrhotic population was performed in four tertiary centres in the USA, but did not include a control group. ${ }^{29}$ These data may differ from European experience with a low proportion of compensated cirrhosis (only $2 \%$ had ChildPugh class A disease), high incidence of hepatitis C $(44 \%)$ and high rates of inpatient dialysis (24\%). We therefore sought to add to these studies by further evaluating the utility of current diagnostic criteria for AKI in patients with cirrhosis and examine their association with patient outcomes.

\section{METHODS}

\section{Setting}

The Royal Derby Hospital is a 1139-bed teaching hospital that provides all major medical and surgical specialities (excepting neuro- and cardiothoracic surgery).
The liver unit is a tertiary referral centre, serving a population of 700000 . There is a central chemical pathology laboratory for all inpatient and outpatient samples. A compensated kinetic Jaffe method with an inter-assay coefficient of variance of $2.3 \%$ at $96 \mu \mathrm{mol} /$ 1 (Roche P-analyser, Roche Diagnostics, West Sussex, UK) was used to measure all serum creatine values throughout the study period (normal creatine range $70-120 \mu \mathrm{mol} / \mathrm{l})$.

\section{Detection of AKI}

We have devised and implemented a real-time electronic reporting system for AKI that identifies all cases of AKI across our hospital. This is based on the AKIN diagnostic criteria, which are summarised in table $1 .^{15}$ This system has been in clinical use since 2010 and its diagnostic accuracy has been established. A full description of the system is available elsewhere. ${ }^{30}$

This system also allows prospective data collection for all cases of AKI at our centre. A daily electronic report of all AKI episodes is automatically generated that details patient location (community vs hospital-acquired), patient age and baseline creatine. These data are supplemented by the highest AKI stage, last serum creatine in stay (to assess renal recovery), length of hospital stay and whether the patient survived to hospital discharge. Comorbidity data (based on ICD-10) coding and Charlson score are also included. Approval to use anonymised data in this way was obtained from the National Information Governance Board and a Local Research Ethics Committee waived requirement of individual patient consent.

\section{Identification of patients with cirrhosis}

All patients under the care of the Gastroenterology Department were selected from the prospectively identified hospital-wide population of those with AKI in the period 1 June 2010-31 October 2011. These patients were then manually screened to identify those with cirrhosis. Cirrhosis was diagnosed on the basis of any clinical (eg, decompensated chronic liver disease), radiological (eg, coarse liver, splenomegaly, presence of varices), or laboratory parameters (eg, prolonged international normalised ratio (INR) low platelets) and/or histological evidence from liver biopsy when available. $^{29} 31$

A control group of patients with cirrhosis without AKI were retrospectively identified from the

Table 1 Classification/staging system for AKI according to AKIN ${ }^{15}$

\begin{tabular}{lll}
\hline AKI stage & Serum creatine criteria & Urine output criteria \\
\hline AKI stage 1 & Increase in serum creatine $\geq 0.3 \mathrm{mg} / \mathrm{dl}$ or increase to $\geq 150 \%-200 \%$ from baseline & Urine output $<0.5 \mathrm{ml} / \mathrm{kg} / \mathrm{h} \mathrm{for}>6 \mathrm{~h}$ \\
AKI stage 2 & Increase of serum creatine to $>200 \%-300 \%$ from baseline & Urine output $<0.5 \mathrm{ml} / \mathrm{kg} / \mathrm{h} \mathrm{for}>12 \mathrm{~h}$ \\
AKI stage 3 & Increase of serum creatine to $>300 \%$ from baseline & Urine output $<0.3 \mathrm{ml} / \mathrm{kg} / \mathrm{h} \mathrm{for} 24 \mathrm{~h}$ \\
& OR serum creatine $\geq 4.0 \mathrm{mg} / \mathrm{dl}$ after a rise of at least $0.5 \mathrm{mg} / \mathrm{dl}$ & OR anuria for $12 \mathrm{~h}$ \\
& OR treatment with renal replacement therapy &
\end{tabular}

AKI, acute kidney injury; AKIN, acute kidney injury network. 
admission register to the liver unit over the same time period. Sequential patients were manually screened for evidence of cirrhosis in the same way as previously detailed. Patients were included only once in the control group regardless of the number of admissions during the 18 -month period. Data were taken exclusively from the initial admission.

Child-Pugh score and class were retrospectively established for all cirrhotic patients included in the study. Where possible, this was calculated from electronic records within 3 months of the acute admission. If not possible, it was established from the results nearest to normal during the admission.

\section{Statistical analysis}

Parametric data are presented as mean \pm SD and nonparametric data as median (inter-quartile range). $\chi^{2}$ Test was used to compare categorical data and t test or Mann-Whitney test to compare continuous data depending on whether data were parametric or nonparametric. A Kaplan-Meier survival curve was calculated based on mortality data censored for admission. Binary logistic regression was used to test significant univariate associations with inhospital mortality. $\mathrm{p}$ Values of $<0.05$ were considered significant. All analyses were performed using SPSS V.19.

\section{RESULTS}

\section{Cirrhotic population}

A total of 162 patients with cirrhosis were included, 110 with AKI and 52 controls. Of the 110 patients with cirrhosis who sustained AKI, 44 patients (40\%) had AKI stage 1, 32 patients stage $2(29.1 \%)$ and 34 $(30.9 \%)$ stage 3 . The mean age of the cirrhotic population was $56.8 \pm 13.6$ years. Patients were predominantly male (65.4\%) and $78.4 \%$ had liver disease caused by alcohol. The remainder of patients had fatty liver disease (6.2\%), cryptogenic cirrhosis (5.5\%), autoimmune hepatitis (3.7\%), Hepatitis C (HCV) (1.9\%), primary biliary cirrhosis (PBC) (1.9\%) and other causes $(2.5 \%)$. A total of 50 patients $(30.9 \%)$ had biopsy-proven cirrhosis; $6.2 \%$ were recorded as having pre-existing chronic kidney disease. The mean baseline creatine was $78.4 \pm 29.4 \mu \mathrm{mol} / \mathrm{l}$.

\section{AKI versus non-AKI in patients with cirrhosis}

Patient characteristics were similar when comparing those with and without AKI, as summarised in table 2. There was no significant difference between ages of the groups (59.6 \pm 13.4 vs $50.9 \pm 12.2, \mathrm{p}=0.842)$, sex $(62.7 \%$ vs $71.2 \%$ male, $\mathrm{p}=0.292)$ or aetiology of liver disease $(79.1 \%$ vs $76.9 \%$ alcohol, $\mathrm{p}=0.754)$. There was also comparable severity of liver disease $(p=0.135$ for trend) and similar comorbidities between the groups. The only differences were the prevalence of diabetes $(29.1 \%$ in the AKI group vs $11.5 \%$ in the control group, $\mathrm{p}=0.014)$ and mean baseline creatine
Table 2 Comparisons between cirrhotic groups: cirrhotics with acute kidney injury (AKI) and cirrhotic controls without AKI

\begin{tabular}{|c|c|c|c|}
\hline Factor & $\begin{array}{l}\text { Cirrhotics } \\
\text { with AKI }\end{array}$ & $\begin{array}{l}\text { Cirrhotic controls } \\
\text { without AKI }\end{array}$ & Significance \\
\hline AKI stage 0 & - & $52(100 \%)$ & \\
\hline AKI stage 1 & $44(40 \%)$ & - & \\
\hline AKI stage 2 & $32(29.1 \%)$ & - & \\
\hline AKI stage 3 & $34(30.9 \%)$ & - & \\
\hline \multicolumn{4}{|c|}{ Severity of liver disease } \\
\hline Mean CPS & 8.87 & 8.29 & $p=0.364$ \\
\hline CPA & $17(15.5 \%)$ & $15(28.8 \%)$ & $p=0.135$ \\
\hline$C P B$ & $52(47.2 \%)$ & $21(40.4 \%)$ & \\
\hline $\mathrm{CPC}$ & $41(37.1 \%)$ & $16(30.8 \%)$ & \\
\hline \multicolumn{4}{|l|}{ Comorbidities } \\
\hline Diabetes & $32(29.1 \%)$ & $6(11.5 \%)$ & $p=0.014$ \\
\hline Lung disease & $9(8.2 \%)$ & $7(13.5 \%)$ & $p=0.293$ \\
\hline IHD & $5(4.5 \%)$ & $0(0 \%)$ & $p=0.118$ \\
\hline Acute MI & $5(4.5 \%)$ & $0(0 \%)$ & $p=0.118$ \\
\hline CCF & $5(4.5 \%)$ & $1(1.9 \%)$ & $p=0.409$ \\
\hline CKD & $8(7.3 \%)$ & $2(3.8 \%)$ & $p=0.398$ \\
\hline Stroke & $4(3.6 \%)$ & $0(0 \%)$ & $p=0.164$ \\
\hline $\begin{array}{l}\text { Connective } \\
\text { tissue disease }\end{array}$ & $2(1.8 \%)$ & $0(0 \%)$ & $p=0.328$ \\
\hline Dementia & $0(0 \%)$ & $1(1.9 \%)$ & $p=0.145$ \\
\hline $\begin{array}{l}\text { Peptic ulcer } \\
\text { disease }\end{array}$ & $3(2.7 \%)$ & $1(1.9 \%)$ & $p=0.758$ \\
\hline $\begin{array}{l}\text { Peripheral } \\
\text { vascular disease }\end{array}$ & $3(2.7 \%)$ & $1(1.9 \%)$ & $p=0.758$ \\
\hline Cancer & $3(2.7 \%)$ & $0(0 \%)$ & $p=0.229$ \\
\hline $\begin{array}{l}\text { Diabetic } \\
\text { complications }\end{array}$ & $0(0 \%)$ & $1(1.9 \%)$ & $p=0.145$ \\
\hline
\end{tabular}

AKI, acute kidney injury; CPS, Child-Pugh score; CP A, Child-Pugh A, B and $C$ etc; IHD, ischaemic heart disease; $\mathrm{MI}$, myocardial infarction; CCF, congested cardiac failure; CKD, chronic kidney disease.

value $(85.5 \pm 28.8 \mu \mathrm{mol} / \mathrm{l}$ in the AKI group vs $64.7 \pm 25.6 \mu \mathrm{mol} / \mathrm{l}$ in the control group, $\mathrm{p}=0.03$ ).

\section{Factors associated with outcomes}

The presence of AKI in the cirrhotic population was associated with significantly increased mortality, $31.8 \%$ versus $3.8 \%$ in the control group $(\mathrm{p}<0.001)$, summarised in figure 1 . We also observed that mortality increased as AKI became more severe, as shown in figure 2. Inhospital mortality rates were $13.5 \%$ in AKI stage $1,37.8 \%$ in AKI stage 2 and $43.2 \%$ in AKI stage $3\left(\chi^{2}\right.$ for trend, $\left.\mathrm{p}<0.001\right)$. A Kaplan-Meier survival curve shows inhospital mortality associated with each stage of AKI (figure 3). This difference was significant between AKI stages 1 and $2(p=0.001)$, but not between AKI stages 2 and $3(p=0.793)$. AKI was also associated with an increased length of stay: median 6.0 days (IQR 4.0-8.75) versus 16.0 days (IQR 6.0-27.5), $\mathrm{p}<0.001$.

There was also an association between mortality and increasing severity of liver disease as measured by Child-Pugh class. Mortality rates were $3.1 \%$ in class $\mathrm{A}, 23.6 \%$ in class $\mathrm{B}$ and $32.8 \%$ in class $\mathrm{C}\left(\chi^{2}\right.$ for trend $=0.006$ ) (figure 4). 


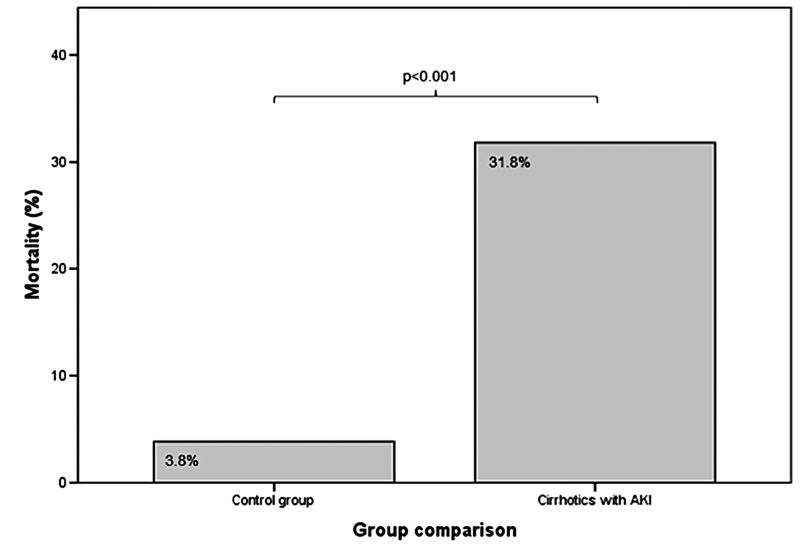

Figure 1 Overall mortality of cirrhotics with and without (control group) acute kidney injury (AKI).

We compared survivors and non-survivors to identify additional factors associated with inhospital mortality. Previous myocardial infarction $(1.6 \%$ vs $8.1 \%$, $\mathrm{p}=0.044)$, peripheral vascular disease $(0.8 \%$ vs $8.1 \%$, $\mathrm{p}=0.012)$, connective tissue disease $(0 \%$ vs $5.4 \%$, $\mathrm{p}=0.009)$ and the presence of pre-existing chronic kidney disease $(\mathrm{CKD})(4 \%$ vs $13.5 \%, \mathrm{p}=0.035)$ were associated with increased mortality. Other comorbid conditions were not associated with death, including diabetes $(21.6 \%$ vs $29.7 \%, \mathrm{p}=0.305)$ and baseline creatine (median $77.8 \pm 31.4$ vs $80.3 \pm 22.0 \mu \mathrm{mol} / \mathrm{l}$, $\mathrm{p}=0.181$ ). In our population, there was no difference in age between survivors and non-survivors (median $55.9 \pm 13.9$ vs $59.8 \pm 12.3$ years, $\mathrm{p}=0.193$ ).

Binary logistic regression including all significant univariate associations revealed that the only factors independently associated with increased mortality were AKI, Child-Pugh class B and Child-Pugh class C (Nagelkerke $r^{2}=0.256$, Hosmer and Lemeshow test for goodness of fit $p=0.567)$. Table 3 shows this model in full.

\section{DISCUSSION}

This study demonstrates that current creatine-based definitions of AKI retain their utility in cirrhotic

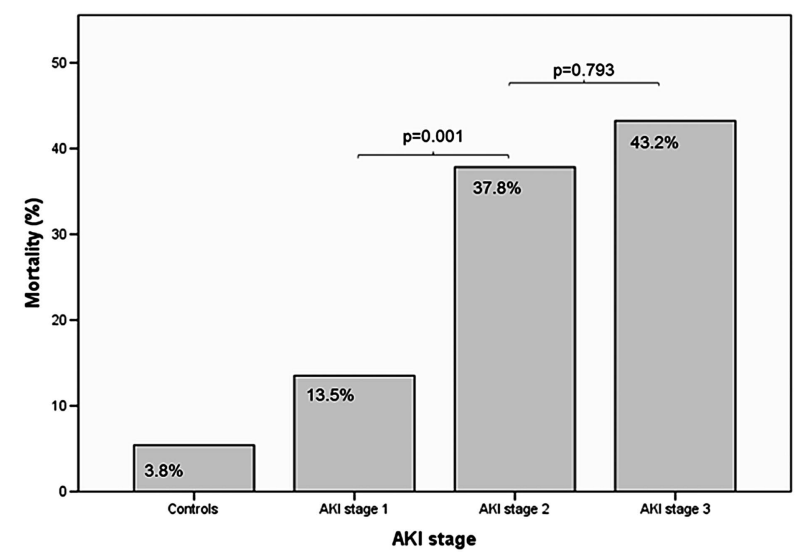

Figure 2 Mortality across acute kidney injury (AKI) stages.

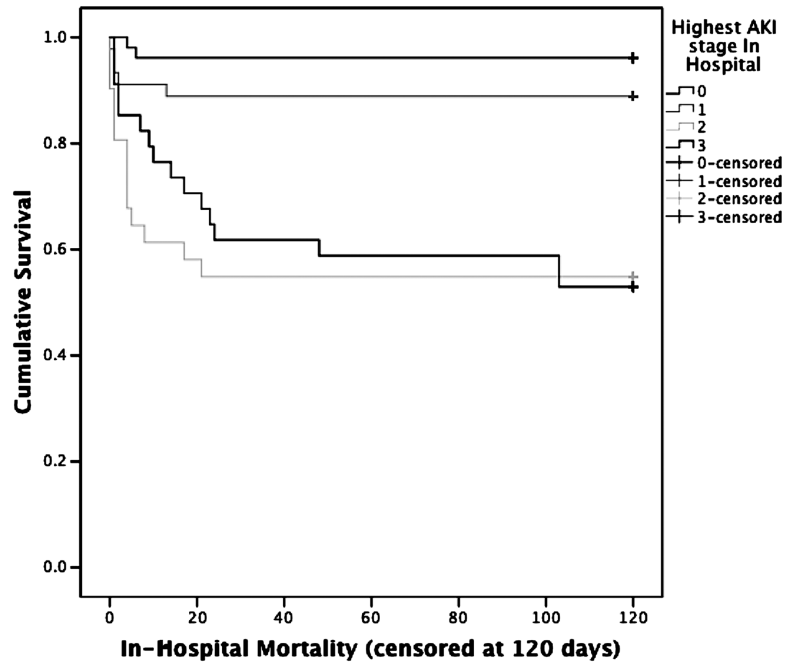

Figure 3 Kaplan-Meier survival curve showing inhospital mortality in cirrhotics with and without acute kidney injury (AKI) (censored at 120 days).

patients, with the presence and increasing severity of AKI strongly associated with inhospital mortality. Our results add to the relatively small number of studies examining the use of such criteria in cirrhotic patients and provide an important comparison between those with and without AKI.

Previous criteria for identifying renal dysfunction, such as the HRS definitions, ${ }^{10}$ use arbitrary creatine thresholds which fail to incorporate an individual's baseline renal function and exclude smaller changes in creatine values despite their impact on patient outcome. Newer AKI criteria, such as the AKIN or KDIGO classifications, have several advantages. Small but significant changes in an individual's baseline creatine are included to improve sensitivity; these criteria also describe the severity of AKI depending on the magnitude of creatine rise (or degree of oliguria). These criteria are now widely accepted and have been validated in a variety of clinical scenarios, ${ }^{14}$ but the differences in creatine profile seen in liver patients

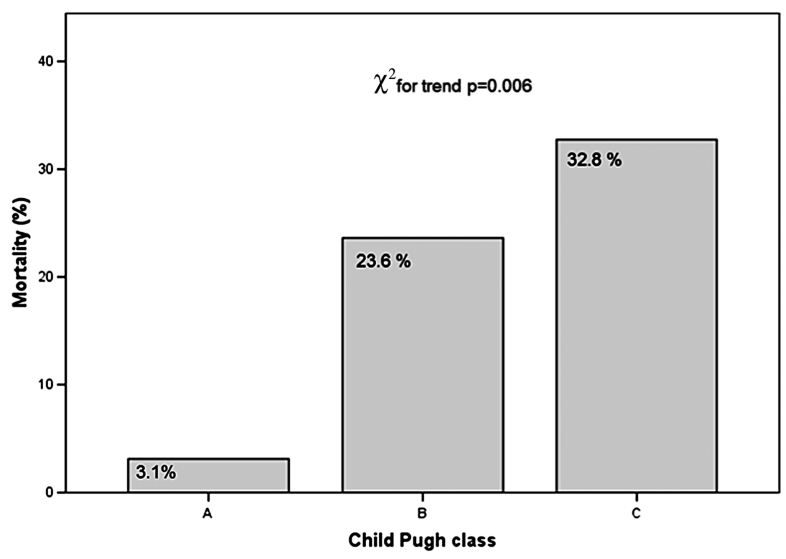

Figure 4 Mortality with increasing severity of liver disease by Child-Pugh class. 
Table 3 Multivariate analysis of factors independently associated with mortality

\begin{tabular}{llrl}
\hline Factor & Significance & HR & $95 \%$ Cls \\
\hline Acute kidney injury & 0.02 & 10.6 & 2.4 to 47.0 \\
Child-Pugh B & 0.04 & 8.1 & 1.0 to 65.4 \\
Child-Pugh C & 0.016 & 13.1 & 1.6 to 106.0 \\
\hline
\end{tabular}

mandate that these criteria are examined specifically in this patient group. To date, there have been a small number of studies that have attempted this. Carvalho et $\mathrm{al}^{32}$ retrospectively studied 91 patients with cirrhosis and ascites identifying AKI within $48 \mathrm{~h}$ of hospital admission. The majority of patients had mild renal dysfunction (91\% had stage 1 AKI). Patients with AKI had a hospital mortality rate of $52.7 \%$. Presence of AKI conferred an OR of 2.6 for hospital mortality but quantifying the risk by stage was limited by the small numbers of patients with more severe AKI. Belcher et $a l^{29}$ prospectively studied 192 hospitalised cirrhotic patients with AKI in four centres in the USA. AKIN criteria were used to identify AKI, with 50 (26\%) patients with AKI stage 1, 47 (24\%) stage 2 and 95 (49\%) stage 3 . Baseline creatine was robustly defined as the most recent stable measurement prior to admission for the index hospitalisation within 1 year or if they had at least 5 days of stable values within the normal creatine range following admission. Overall mortality was 26\% and severity of AKI was independently associated with mortality (adjusted OR=3.8, 95\% CI 1.3 to 11.1), but the presence of AKI itself was not assessed as there was no control group without AKI. Tsient et al ${ }^{31}$ prospectively studied a total of 91 patients with cirrhosis and ascites recruited from hepatology outpatient clinics. Patients had bloods performed at 4-6 weekly intervals and AKI was defined by AKIN criteria if two samples within $48 \mathrm{~h}$ were available or if a $50 \%$ increase in serum creatine from the baseline visit was observed. If identified, AKI was treated with volume expansion of albumin $(1 \mathrm{~g} / \mathrm{kg})$ for two consecutive days. In all, 49 patients suffered with 82 episodes of AKI, and again mortality was higher in those with AKI.

Our results add to these studies and confirm that AKI (as per AKIN criteria) is associated with mortality in patients with cirrhosis, even after adjustment for severity of liver disease and comorbid conditions. Although comparisons between studies are difficult, mortality rates in cirrhotics with AKI do appear to be higher than other reports of generalised hospital patients with AKI, suggesting that this patient group is particularly vulnerable when AKI develops. ${ }^{17} 30$ For contextual reference, the overall mortality of general hospitalised patients with AKI at our centre from September 2010 to August 2012 was 21.2\% (stage 1, 15.2\%; stage 2, 32.3\%; stage 3, 33.5\%). Despite potential differences between the demographics of USA and European cirrhotics, we observed mortality rates similar to those reported by Belcher et al. ${ }^{29}$ Although high (at over 30\%), our observed mortality rates are lower than some historic studies. ${ }^{26} 27$ This may reflect our inclusion of patients with less severe AKI, an effect of using current diagnostic criteria; mortality rose to above $40 \%$ in those with AKI stage 3. However, it is important to recognise that even small acute rises in serum creatine are significant, with mortality in our patients with AKI stage 1 substantially elevated as compared with controls. The implications of relatively small changes in serum creatine often remain underappreciated in clinical practice, which may be partly addressed by wider usage of current AKI definitions.

Our observed mortality rate in the control group was low (3.8\%). It is, however, similar to that reported in other studies of cirrhotic patients with ascites who did not sustain AKI. ${ }^{31}$ This may reflect the relatively young age of patients with cirrhosis, the low prevalence of comorbidity or a less severe acute illness without factors that may precipitate AKI.

Comparison between the groups with and without AKI showed they were well matched in terms of age, sex, cause and severity of liver disease. There were few differences in renal risk profile between the control group and those with cirrhosis and AKI, with the only differences in baseline creatine and diabetes. Although diabetes was not associated with increased mortality, it is possible that its presence may be a risk factor for developing AKI. The higher baseline creatine may reflect a gradual increase in creatine which may occur as a prodrome of renal dysfunction in patients with cirrhosis. ${ }^{33}$ Particular attention should therefore be paid defining baseline creatine in this group. In general patients, avoiding the use of results from the 7 days prior to hospital admission may avoid the use of falsely high reference creatine values, ${ }^{34}$ but in cirrhotic patients it is conceivable that this period should be extended due to the indolent increase sometimes in serum creatine prior to the acute presentation.

The modified Child-Turcotte-Pugh score is widely used as a simple descriptive or prognostic indicator in patients with cirrhosis. ${ }^{35}{ }^{36}$ Unlike the model of endstage liver disease score ${ }^{37}$ it does not include a marker of renal dysfunction, although it could be argued that the inclusion of bilirubin, ascites and encephalopathy are potential surrogate markers of renal injury. ${ }^{38}$ It is not surprising that increasing mortality was associated with increasing severity of liver disease in a stagedependent manner as measured by the Child-Pugh class. It does, however, support that the scores were credibly calculated with a valid methodology.

Our study does have some potential weaknesses. The methodology for electronic identification of AKI employed by this study has been previously validated in a generalised hospital population with a false negative rate of $0.2 \% .{ }^{30}$ However, in a population of patients with cirrhosis who are younger with lower than average baseline serum creatine values, it is possible that the initial step, which depends on a comparison with an estimated creatine value, may miss a small number of cases of AKI stage 1. In addition, the data presented are limited to 
information collected routinely as standard of care either in patient notes or on electronic record. Comorbidity data are based upon International Classification of Diseases - version 2010 (ICD-10) coding and therefore limited to these definitions. Thus, we are unable to report the impact of other variables, such as circulatory dysfunction, infection or inflammation. Further studies could evaluate whether changing AKIN creatine thresholds would be of added benefit in cirrhotics.

In conclusion, this study shows that AKI, as defined by the AKIN diagnostic criteria, is associated with increased mortality in patients with cirrhosis in a stagedependent manner. Severe liver disease and AKI appear to be independent variables in predicting death in cirrhotic patients. The current methods for defining AKI appear to have clinical utility in patients with cirrhosis, and their wider use may help with improved recognition and earlier intervention for AKI, which has particular relevance in this vulnerable group.

\section{What is already known about this subject}

- Renal dysfunction in cirrhosis is common and confers a poor prognosis.

- Previous definitions of renal dysfunction in patients with cirrhosis (eg, HRS definitions) have relied on creatinine thresholds.

- AKIN criteria recognise that a small decline in renal function is associated with poor outcomes in general hospitalised patients.

\section{What this study adds}

AKI, as defined by the AKIN diagnostic criteria, is associated with increased mortality in patients with cirrhosis in a stage-dependent manner.

- Mortality rates in cirrhotics with AKI appear to be higher than other reports of generalised hospital patients with AKI.

- Severe liver disease and AKI appear to be independent variables in predicting death in cirrhotic patients.

How might it impact on clinical practice in the foreseeable future

AKIN criteria appear to have clinical utility in patients with cirrhosis.

- Their wider use may help with improved recognition and earlier intervention for AKI which should improve patient outcomes.

- Further work is needed to understand the mechanism and risk factors in developing renal dysfunction in patients with cirrhosis.
Contributors NS and AA conceived the study and overall supervised conduct of the study. RS and NS contributed to study design, data collection, analysis and interpretation. NK contributed to data collection. CM contributed to data interpretation. All authors contributed to the drafting, revising of the article and approved the final version of the manuscript.

Competing interests None.

Ethics approval National Information Governance Board.

Provenance and peer review Not commissioned; externally peer reviewed.

Open Access This is an Open Access article distributed in accordance with the Creative Commons Attribution Non Commercial (CC BY-NC 3.0) license, which permits others to distribute, remix, adapt, build upon this work non-commercially, and license their derivative works on different terms, provided the original work is properly cited and the use is non-

commercial. See: http://creativecommons.org/licenses/by-nc/3.0/

\section{REFERENCES}

1 WHO Organization. WHO European Health for All database. 2012. http://www.euro.who.int/en/what-we-do/

data-and-evidence/databases/european-health-for-all-databasehfa-db2 (accessed 2 Dec 2012).

2 Sheron N. A time to act: Improving liver health and outcomes in liver disease. London: British Society of Gastroenterology, 2009.

3 National End of Life Care Intelligence Network. Deaths from Liver Disease: Implications for end of life care in England, 2012. http://www.endoflifecare-intelligence.org.uk/resources/ publications/deaths_from_liver_disease (accessed 9 April 2013).

4 du Cheyron D, Bouchet B, Parienti J, et al. The attributable mortality of acute renal failure in critically ill patients with liver cirrhosis. Intensive Care Med 2005;31:1693-9.

5 Garcia-Tsao G, Parikh CR, Viola A. Acute kidney injury in cirrhosis. Hepatology 2008;48:2064-77.

6 D’Amico G, Garcia-Tsao G, Pagliaro L. Natural history and prognostic indicators of survival in cirrhosis: a systematic review of 118 studies. J Hepatol 2006;44:217-31.

7 Ginés P, Schrier RW. Renal failure in cirrhosis. N Engl J Med 2009;361:1279-90.

8 Alessandria C, Ozdogan O, Guevara M, et al. MELD score and clinical type predict prognosis in hepatorenal syndrome: relevance to liver transplantation. Hepatology 2005;41:1282-9.

9 Al Sibae MR, Cappell MS. Accuracy of MELD scores in predicting mortality in decompensated cirrhosis from variceal bleeding, hepatorenal syndrome, alcoholic hepatitis, or acute liver failure as well as mortality after non-transplant surgery or TIPS. Dig Dis Sci 2011;56:977-87.

10 Arroyo V, Ginès P, Gerbes AL, et al. Definition and diagnostic criteria of refractory ascites and hepatorenal syndrome in cirrhosis: International Ascites Club. Hepatology 1996;23:164-76.

11 Chen YC, Tsai MH, Ho YP, et al. Comparison of the severity of illness scoring systmes for critically ill cirrhotic patients with renal failure. Clin Nephrol 2004;61:111-18.

12 Fang JT, Tsai MH, Tian YC, et al. Outcome predictors and new score of critically ill cirrhotic patients with acute renal failure. Nephrol Dial Transp 2008;23:1961-9.

13 Cárdenas A, Ginès P, Uriz J, et al. Renal failure after upper gastrointestinal bleeding in cirrhosis: incidence, clinical course, predictive factors, and short-term prognosis. Hepatology 2001;34:671-6. 
14 Balasubramanian G, Al-ALy Z, Moiz A, et al. Early Nephrologist involvement in Hospital-Acquired Acute Kidney Injury: a pilot study. Am J Kidney Dis 2011;57:228-34.

15 Mehta RL, Kellum JA, Shah SV, et al. Acute Kidney Injury Network. Acute Kidney Injury Network: report of an initiative to improve outcomes in acute kidney injury. Crit Care 2007;11:R31.

16 Bellomo R, Ronco C, Kellum JA, et al. Acute Dialysis Quality Initiative workgroup. Acute renal failure-definition, outcome measures, animal models, fluid therapy and information technology needs: the Second International Consensus Conference of the Acute Dialysis Quality Initiative (ADQI) Group. Crit Care 2004;8:R204-12.

17 Uchino S, Bellomo R, Goldsmith D, et al. An assessment of the RIFLE criteria for acute renal failure in hospitalized patients. Crit Care Med 2006;34:1913-7.

18 Coca SG, Yusuf B, Shlipak MG, et al. Long-term risk of mortality and other adverse outcomes agter acute kidney injury: a systematic review and meta-analysis. Am J Kidney Dis 2009;53:961-73.

19 Lafrance JP, Miller DR. Acute kidney injury associates with increased long-term mortality. J Am Soc Nephrology 2009;21:345-52.

20 Uchino S, Kellum JA, Bellomo R, et al. Beginning and Ending Supportive Therapy for the Kidney (BEST Kidney) Investigators. Acute renal failure in critically ill patients: a multinational, multicenter study. JAMA 2005;294:813-18.

21 Ricci Z, Cruz D, Ronco C. The RIFLE criteria and mortality in acute kidney injury: a systematic review. Kidney Int 2008;73:538-46.

22 Chertow GM, Burdick E, Honour M, et al. Acute kidney injury, mortality, length of stay, and costs in hospitalized patients. J Am Soc Nephrology 2005;16:3365-70.

23 Orlando R, Floreani M, Padrini R, et al. Evaluation of measured and calculated creatinine clearances as glomerular filtration markers in different stages of liver cirrhosis. Clin Nephrol 1999;51:341-7.

24 Dimeski G, McWhinney B, Jones B, et al. Extent of bilirubin interference with Beckman creatinine methods. Ann Clin Biochem 2008;45:91-2.
25 Sherman DS, Fish DN, Teitelbaum I. Assessing renal function in cirrhotic patients: problems and pitfalls. Am J Kidney Dis 2003;41:269-78.

26 Jenq CC, Tsai MH, Tian YC, et al. RIFLE classification can predict short-term prognosis in critically ill cirrhotic patients. Intensive Care Med 2007;33:1921-30.

27 Cholongitas E, Calvaruso V, Senzolo M, et al. RIFLE classification as predictive factor of mortality in patinets with cirrhosis admitted to intensive care unit. J Gastroenterol Hepatol 2009;24: 1639-47.

28 Lopes JA, Melo MJ, Costa AC, et al. Acute kidney injury and in-hospital mortality in critically ill patients with cirrhosis: a cohort study. Gut 2012;61:655-6.

29 Belcher JM, Garcia-Tsao G, Sanyal AJ, et al. TRIBE-AKI Consortium. Association of AKI with mortality and complications in hospitalized patients with cirrhosis. Hepatology 2012;57:753-62.

30 Selby NM, Crowley L, Fluck RJ, et al. Use of electronic results reporting to diagnose and montior AKI in hospitalized patients. Clin J Am Soc Nephrol 2012;7:533-40.

31 Tsien CD, Rabie R, Wong F. Acute kidney injury in decompensated cirrhosis. Gut 2012;62:131-7.

32 de Carvalho JR, Villela-Nogueira CA, Luiz RR, et al. Acute kidney injury network criteria as a predictor of hospital mortality in cirrhotic patients wih ascites. J Clin Gastroenterol, 2012;46:e21-6.

33 Wong F, Nadim M, Kellum J, et al. Working party proposal for a revised classification system of renal dysfunction in patients with cirrhosis. Gut 2011;60:702-9.

34 Siew E, Ikizler T, Matheny M, et al. Estimating baseline kidney function in hospitalized patients with impaired kidney function. Clin J Am Soc Nephron 2012;7:712-9.

35 Child C, Turcotte J. Surgery and portal hypertension. In: Child Ced. The liver and portal hypertension. Philadelphia: W. B. Saunders Co., 1964;1:1-85.

36 Pugh R, Murray-Lyon I, Dawson J, et al. Transection of the oesophagus for bleeding oesophageal varices. Br J Surg 1973;60:646-9.

37 Malinchoc M, Kamath P, Gordon F, et al. A model to predict poor survival in patients undergoing transjugular intrahepatic portosystemic shunts. Hepatology 2000;31:864-71.

38 Durand F, Valla D. Assessment of the prognosis of cirrhosis: child-pugh versus MELD. J Hep 2005;42:S100-107. 